\title{
Piezoelectric Energy Harvesting from Raised Crosswalk Devices
}

\author{
Dario Ticali ${ }^{\mathrm{a}}$ and Mario Denaro ${ }^{\mathrm{b}}$ and Alessandro Barracco ${ }^{\mathrm{c}}$ and Marco Guerrieri ${ }^{\mathrm{d}}$ \\ ${ }^{a, b, c, d}$ Faculty of Engineering and Architecture, \\ University of Enna "Kore", Cittadella universitaria, Enna, Italy
}

\begin{abstract}
This paper presents the main characteristics of an experimental energy harvesting device that can be used to recover energy from the vehicular and pedestrian traffic. The use of a piezoelectric bender devices leads to a innovative approach to Henergy Harvesting. The study focuses on the definition and specification of a mechanical configuration able to transfer the vibration from the main box to the piezoelectric transducer.

The piezoelectric devices tested is the commonly used monolithic piezoceramic material lead-zirconate-titanate (PZT). The experimental results estimate the efficiency of this device tested and identify the feasibility of their use in real world applications. The results presented in this paper show the potential of piezoelectric materials for use in power harvesting applications.
\end{abstract}

Keywords: Piezoelectric; Energy Harvesting; Finite Element Method; Transient analysis; Electric Circuit. PACS: 77. Dielectrics, piezoelectrics, and ferroelectrics and their properties

\section{INTRODUCTION}

The environment is rich in energy sources that, if properly exploited, can make a good contribution to the energetic demand. Clean and economic energy can be extracted from nature using more modern and efficient technologies have allowed to extracted; such tools are able to integrate the production of conventional power plants, or oven to make whole energetically independent units. The research on "energy harvesting", sometimes referred to as "power harvesting" or "energy scavenging" places in this technological progress point of view leading to the optimum and intelligent use of energetical sources.

\section{ENERGY HARVESTING OVERVIEW AND CIRCUIT MODEL}

The term "energy harvesting“ $[4,6,7,10]$ means a process wherby energy derived from alternative sources is captured and exploited: sources usually available in the environment, which represent the alternative energy sources, are converted directly into electrical energy used by specific devices called "energy harvesters". This process does not stop with electronic applications [5], but incorporates numerous fields. Although often refers to applications for low power [9], generally it comes to energy harvesting wherever it is possible to extract energy from a source, by studying and development of a technology able to perform this service [8,11]. The electro-mechanical system under analysis is a rectangular bimorph piezoelectric transuder bonded on a steel cantilever beam.

TABLE 1. Electro-mechanical parameters

\begin{tabular}{cccc}
\hline Symbol & Quantity & Value & Units \\
\hline $\mathrm{Lp}$ & Piezo length & 40,00 & $\mathrm{~mm}$ \\
$\mathrm{Wp}$ & Piezo width & 16,00 & $\mathrm{~mm}$ \\
$\mathrm{Tp}$ & Piezo thickness & 0,80 & $\mathrm{~mm}$ \\
$\mathrm{Tpu}$ & Upper patch thickness & 0,36 & $\mathrm{~mm}$ \\
$\mathrm{Tpl}$ & Lower patch thickness & 0,36 & $\mathrm{~mm}$ \\
$\mathrm{Tsp}$ & Steel lamina thickness & 0,08 & $\mathrm{~mm}$ \\
$\mathrm{~K}$ & Elastic constant & 30,80 & $\mathrm{~N} / \mathrm{mm}$ \\
\hline
\end{tabular}

This converter is made of three layers: the first two are the external piezo (the active layers) while the third layer is the center thin metallic lamina (the passive layer). The latter layer is a connection between the two active layers; furthermore it distances those two layers from the neural axis, so increasing strains and thus electric potential. The converter is made of Lead Zirconate Titanate (PZT-5A): such material has good strength and high electric output (see Table 1). In the present paper both the parallel and the serial configurations for the poling direction are analized. 
The Energy Harvesting device is designed to be installed inside a raised crosswalk with a hollow space in order to feed the road marker cat-eyes, useful for detecting the presence of obstacles such as roundabouts and CUSPIDI on the roadway or for signaling the presence of pedestrians [1].(see Figure 1).

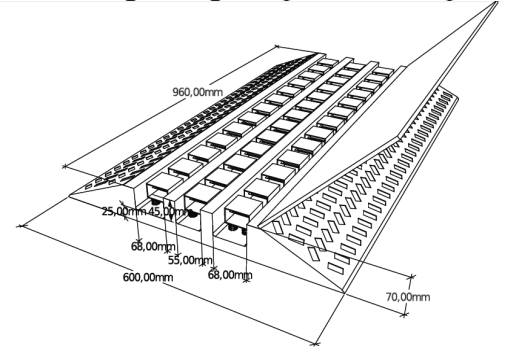

FIGURE 1. Raised crosswalk device.
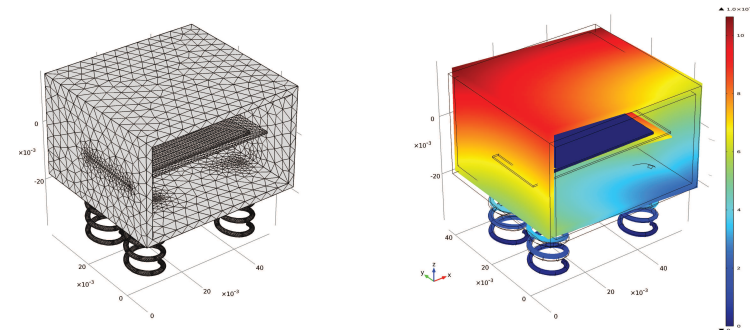

FIGURE 2. FEM model and dynamic solution.

The cantilever piezoelectric transducer under an external load changes its initial state and is subjected to a tensile and compressive load. This resulting, by electromechanical coupling, in electric potential which, using an electric circuit, permits to extract electrical power [2]. This potential difference generated at the extreme poles, via an electrical circuit, is converted into electrical energy. Two different simulations were conducted to evaluate how the response varies, both in terms of electric potential generated electricity for electromechanical coupling. The analysis is developed building a 3D numerical model using COMSOL MULTIPHYSICS 4.2 and QUCS 0.0.16. for the electromechanic coupling and simulation (see Figure 2). The FEM model consists of a free tetrahedric mesh made by 14.658 solid elements and 90.514 dof]. First a static analysis was made by applying a displacement imposed to the free end of $0.1 \mathrm{~mm}$ and secondly two dynamical studies were made respectively with and without the circuit.

\section{ELECTRO-MECHANICAL MODEL}

The piezoelectric generator is optimally suitable for the application above said, both to the small size of the generator itself, which for the vibration system to which the generator is subjected. The energy obtained from the piezoelectric transducers, is not directly usable by electronic devices, for random variations in power and voltage over time. The general structure of the power manager circuit may be divided into three interfaces, as shown in Figure 3. The output voltage from a piezoelectric generator is usually characterized by a periodic behavior and assumes alternately positive and negative values. It is therefore always necessary to a rectifier circuit.

The circuit uses a small piezoelectric transducer to convert mechanical vibration into a source of AC voltage that is fed to the rectifier bridge internal dell'LTC3588. It can recover small vibrations and generate power instead of using traditional batteries [3].

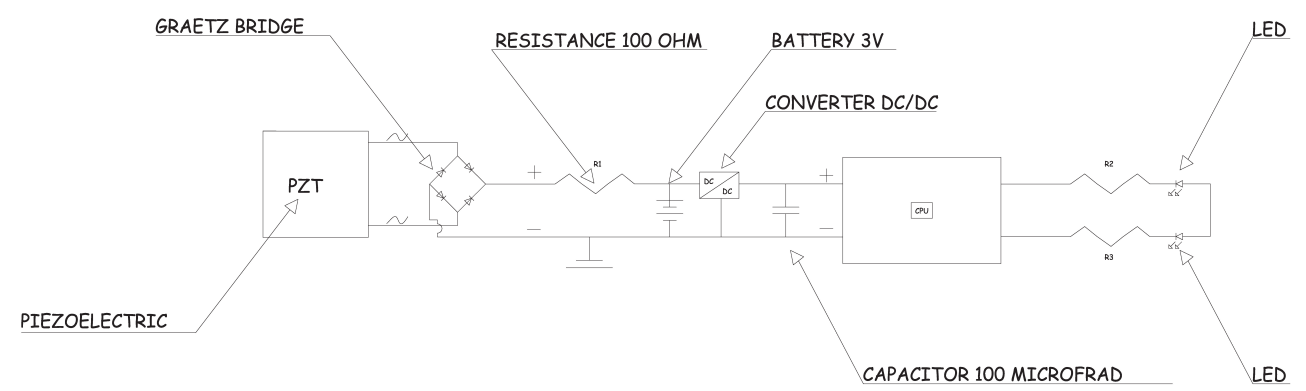

FIGURE 3. The complete electro-mechanical system proposed.

\section{NUMERICAL RESULTS AND DISCUSSION}

System simulations are done using two different configurations in order to evaluate how the model response changes as the poling direction and the external load varies, both in terms of electric potential that electric energy generated for electromechanical coupling. As mentioned above, the system of energy harvesting realized, subjected to an external pulse undergoes a tensile stress to half of the fibers that compose and compression for the remaining half, which translates, for electromechanical coupling, in electric potential (Figure 4). 


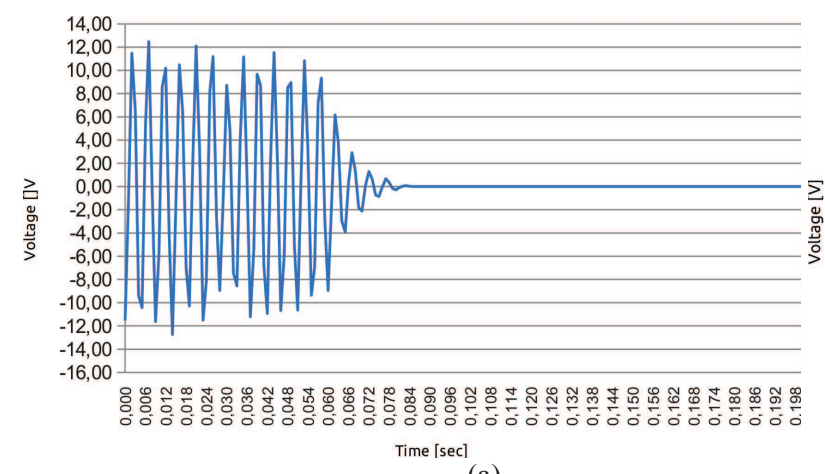

(a)

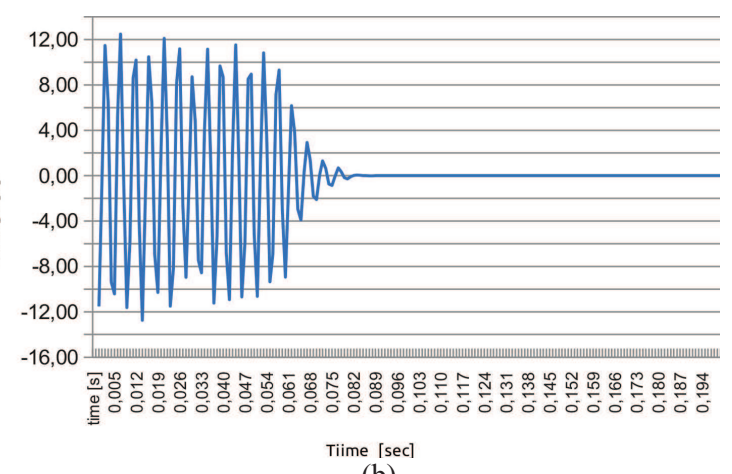

(b)

FIGURE 4. Electric voltage vs time. a) Serial configuration; b) Parallel configuration

This potential difference generated at the extreme poles, through an electrical circuit, is converted into electrical energy. Simulations shows that increasing the R2 resistance value (Figure 5) results in a more time-stable load voltage, both for the serial and the parallel configuration (Figure 6).

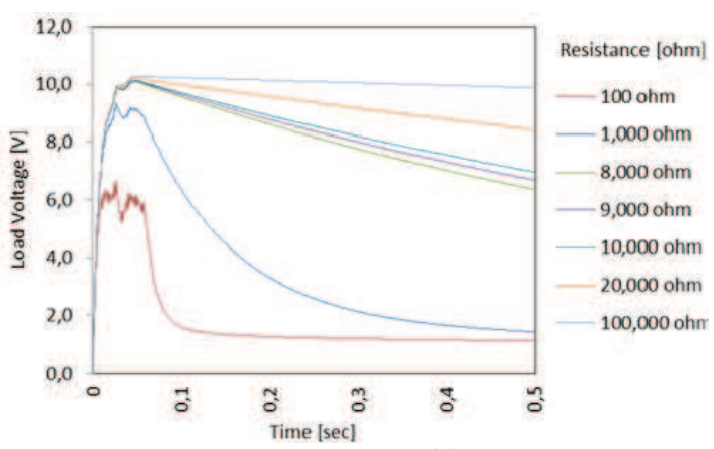

(a)

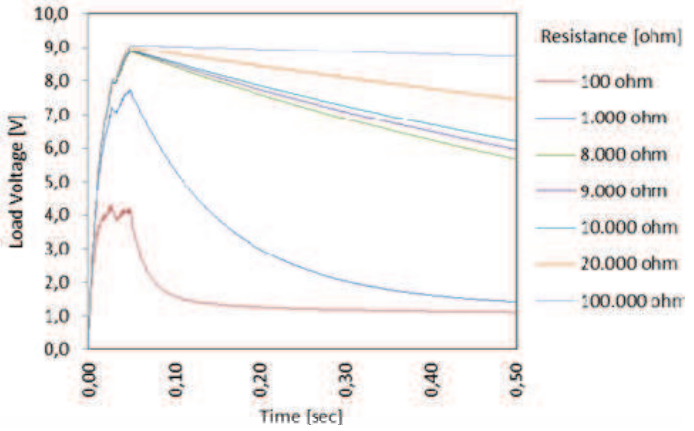

(b)

FIGURE 5. Electric voltage vs time for different resistance values: (a) Serial configuration (b) Parallel configuration.

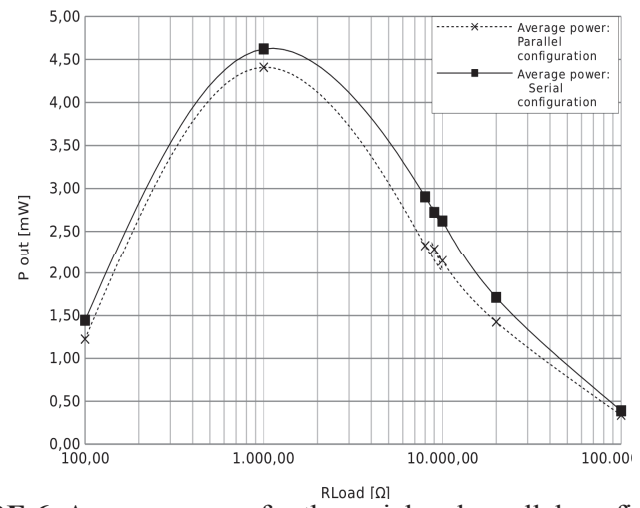

FIGURE 6. Average power for the serial and parallel configuration.

By comparing the peak voltages measured in the parallel configuration (Table 2) and in series configuration (Table 3), is a slight predominance for the latter.

\section{CONCLUSIONS}

The research was based on the study of piezoelectric materials applied to transport infrastructure aimed at producing electric power generated by vehicular and pedestrian traffic. 
TABLE 2. Average voltage and Harvested Power vs resistance: Serial configuration.

\begin{tabular}{ccccccccc}
\hline Rload & {$[\Omega]$} & $1 \mathrm{e}+02$ & $1 \mathrm{e}+03$ & $8 \mathrm{e}+03$ & $9 \mathrm{e}+03$ & $1 \mathrm{e}+04$ & $2 \mathrm{e}+04$ & $1 \mathrm{e}+05$ \\
$\mathrm{Vm}$ & {$[\mathrm{V}]$} & 0,38 & 2,15 & 4,82 & 4,95 & 5,12 & 5,85 & 6,23 \\
$\mathrm{P}$ & {$[\mathrm{mW}]$} & 1,44 & 4,62 & 2,90 & 2,72 & 2,62 & 1,71 & 0,29 \\
\hline
\end{tabular}

TABLE 3. Average voltage and Harvested Power vs resistance: Parallel configuration.

\begin{tabular}{ccrrrrrrr}
\hline R load & $\Omega$ & $1 \mathrm{e}+02$ & $1 \mathrm{e}+03$ & $8 \mathrm{e}+03$ & $9 \mathrm{e}+03$ & $1 \mathrm{e}+04$ & $2 \mathrm{e}+04$ & $1 \mathrm{e}+05$ \\
$\mathrm{Vm}$ & {$[\mathrm{V}]$} & 0,35 & 2,10 & 4,32 & 4,54 & 4,54 & 4,63 & 5,34 \\
$\mathrm{P}$ & {$[\mathrm{mW}]$} & 1,23 & 4,41 & 2,33 & 2,29 & 2,14 & 1,43 & 0,34 \\
\hline
\end{tabular}

In the study, it is characterized both in terms of electric potential that of energy produced, the response of a piezoelectric transducer bender, subjected to an external pulse (vehicular and pedestrian). System simulations are done using two different configurations in order to evaluate how the model response changes as the poling direction and the external load varies. The analysis is developed building a 3D numerical model using COMSOL MULTIPHYSICS 4.2 and QUCS 0.0 .16 for the electromechanic coupling and simulation. The positive results obtained by the numerical analysis above, have led to experimental applications. The experimental phase involved the installation of an energy harvesting device inside a step pedestrian floor, with cavity, with the aim of feeding the road marker such as "cat eyes" adopted for road safety and useful for the detection of the presence of obstacles such as cusps and roundabouts on the road.

\section{REFERENCES}

1. U. K. Singh and R. H. Middleton, "Piezoelectric power scavenging of mechanical vibration energy", Australian Mining Technology Conference, 2-4 October (2007), pages 111-118.Y.

2. C. Shu and I. C. Lien, "Analysis of power output for piezoelectric energy harvesting systems", Smart Materials and Structures 15 (2006), pages 1499-1512.

3. Henry A. Sodano and Daniel J. Inman. "Comparison of Piezoelectric Energy Harvesting Devices for Recharging Batteries", LA-UR-04-5720, Journal of Intelligent Material Systems and Structures, 16(10), 799-807, 2005

4. Collotta, M., Pau, G., Salerno, V.M., Scata, G. "A fuzzy based algorithm to manage power consumption in industrial Wireless Sensor Networks”, (2011) IEEE International Conference on Industrial Informatics (INDIN), art. no. 6034854, pp. 151-156.

5. Collotta, M., Pau, G., Caponetto, R. "A Real-Time System based on a Neural Network Model to Control Hexacopter Trajectories", (2014) International Symposium on Power Electronics, Electrical Drives, Automation and Motion, pp. 225-230.

6. Collotta, M., Messineo, A., Nicolosi, G., Pau, G. "A self-powered bluetooth network for intelligent traffic light junction management", (2014) WSEAS Transactions on Information Science and Applications, 11, pp. 12-23.

7. Collotta, M., Pau, G., Scatà, G., Campisi, T. "A dynamic traffic light management system based on wireless sensor networks for the reduction of the red-light running phenomenon", (2014) Transport and Telecommunication, 15 (1), pp. 1-11.

8. Collotta, M., Cascio, A.L., Pau, G., Scatá, G. "A fuzzy controller to improve CSMA/CA performance in IEEE 802.15.4 industrial wireless sensor networks", (2013) IEEE International Conference on Emerging Technologies and Factory Automation, ETFA, art. no. 6648094.

9. Collotta, M., Nicolosi, G., Toscano, E., Mirabella, O. “A ZigBee-based network for home heating control”, (2008) Proceedings - 34th Annual Conference of the IEEE Industrial Electronics Society, IECON 2008, art. no. 4758389, pp. 2724-2729.

10. Collotta, M., Messineo, A., Nicolosi, G., Pau, G. “A Dynamic Fuzzy Controller To Meet Thermal Comfort By Using Neural Network Forecasted Parameters as the Input”, (2014) Energies, vol.7, pp. 4727-4756.

11. Collotta, M., Pau, G., Salerno, V.M., Scatà, G. "A novel road monitoring approach using Wireless Sensor Networks", (2012) Proceedings - 2012 6th International Conference on Complex, Intelligent, and Software Intensive Systems, CISIS 2012, art. no. 6245667 , pp. $376-381$. 